\title{
NRG1-Fc improves metabolic health via dual hepatic and central action
}

\author{
Peng Zhang, ${ }^{1}$ Henry Kuang, ${ }^{1}$ Yanlin He, ${ }^{2}$ Sharon O. Idiga, ${ }^{3}$ Siming Li, ${ }^{1}$ Zhimin Chen, ${ }^{1}$ Zhao Yang, ${ }^{4}$ \\ Xing Cai, ${ }^{2}$ Kezhong Zhang, ${ }^{4}$ Matthew J. Potthoff, ${ }^{3}$ Yong $\mathrm{Xu},{ }^{2}$ and Jiandie D. Lin ${ }^{1}$ \\ 'Life Sciences Institute and Department of Cell \& Developmental Biology, University of Michigan Medical Center, \\ Ann Arbor, Michigan, USA. ${ }^{2}$ Children's Nutrition Research Center, Department of Pediatrics and Department of Molecular \\ and Cellular Biology, Baylor College of Medicine, Houston, Texas, USA. ${ }^{3}$ Department of Pharmacology and Fraternal Order \\ of Eagles Diabetes Research Center, University of lowa Carver College of Medicine, lowa City, lowa, USA. \\ ${ }^{4}$ Center for Molecular Medicine and Genetics, Department of Immunology and Biochemistry, Wayne State University \\ School of Medicine, Detroit, Michigan, USA.
}

Neuregulins (NRGs) are emerging as an important family of signaling ligands that regulate glucose and lipid homeostasis. NRG1 lowers blood glucose levels in obese mice, whereas the brown fat-enriched secreted factor NRG4 protects mice from high-fat diet-induced insulin resistance and hepatic steatosis. However, the therapeutic potential of NRGs remains elusive, given the poor plasma half-life of the native ligands. Here, we engineered a fusion protein using human NRG1 and the Fc domain of human IgG1 (NRG1-Fc) that exhibited extended half-life in circulation and improved potency in receptor signaling. We evaluated its efficacy in improving metabolic parameters and dissected the mechanisms of action. NRG1-Fc treatment triggered potent AKT activation in the liver, lowered blood glucose, improved insulin sensitivity, and suppressed food intake in obese mice. NRG1-Fc acted as a potent secretagogue for the metabolic hormone FGF21; however, the latter was largely dispensable for its metabolic effects. NRG1-Fc directly targeted the hypothalamic POMC neurons to promote membrane depolarization and increase firing rate. Together, NRC1-Fc exhibits improved pharmacokinetic properties and exerts metabolic benefits through dual inhibition of hepatic gluconeogenesis and caloric intake.

Authorship note: PZ and HK contributed equally to this work.

Conflict of interest: The authors have declared that no conflict of interest exists.

Submitted: November 8, 2017 Accepted: February 1, 2018 Published: March 8, 2018

Reference information: JCI Insight. 2018;3(5):e98522. https:// doi.org/10.1172/jci.insight. 98522.

\section{Introduction}

The neuregulin (NRG) family of ligands and their cognate ErbB receptor tyrosine kinases play an important role in the regulation of tissue development, growth, and homeostasis (1-3). NRGs are synthesized as membrane-bound precursor proteins, some of which undergo proteolytic cleavage to release the biologically active fragments that act via paracrine and/or endocrine mechanisms. Previous genetic studies have revealed associations between SNPs in the NRG/ErbB signaling pathways and diverse metabolic traits. A multiethnic genome-wide association study identified a SNP at the ErbB4 locus to be strongly associated with greater body mass index in the African American population (4). Two NRG3 gene SNPs were found to be associated with basal metabolic rate and body mass index (5). As such, altered NRG/ ErbB signaling may contribute to the pathogenesis of metabolic disorders in humans.

Recent work has strongly implicated NRGs as endocrine regulators of metabolic physiology. Nrg4, a ligand for ErbB3 and ErbB4, was recently identified as a brown fat-enriched secreted factor that acts on the liver to attenuate de novo lipogenesis and enhance fatty acid $\beta$-oxidation (6-10). Adipose tissue expression of $\mathrm{Nrg} 4$ was downregulated in mouse and human obesity. In fact, serum NRG4 concentrations are inversely associated with nonalcoholic fatty liver disease, metabolic syndrome in obese Chinese adults, and subclinical cardiovascular disease in obese adults (11-13). Mice lacking Nrg4 were more prone to diet-induced insulin resistance and hepatic steatosis, whereas fat-specific Nrg4-transgenic mice were protected from these obesity-associated metabolic disorders (9). Further, the Nrg4/ErbB4 signaling pathway protects hepatocytes from stress-induced cell death, thereby serving as an endocrine checkpoint for the steatosis to steatohepatitis transition (14).

The EGF-like domain of NRG1 has been previously demonstrated to promote glucose uptake and mitochondrial oxidative metabolism in cultured myotubes (15-17). When administered in mice, this frag- 
A

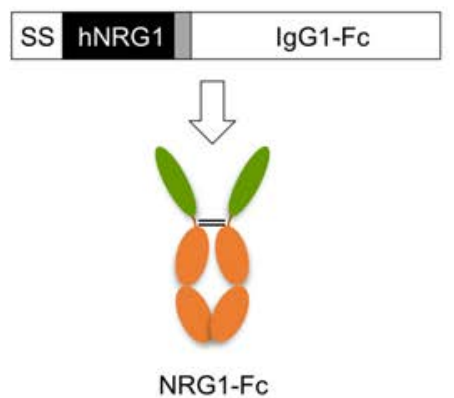

C

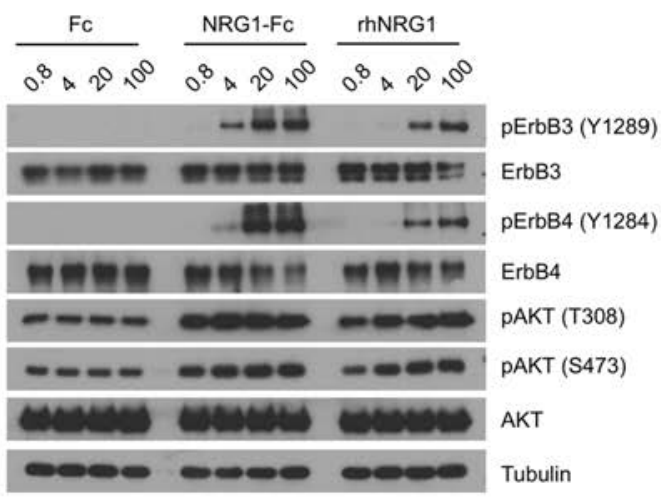

D

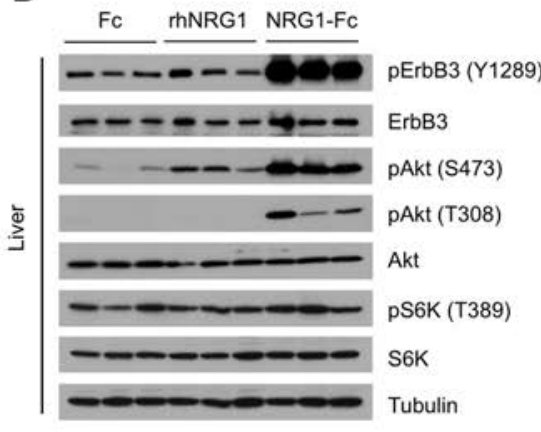

B

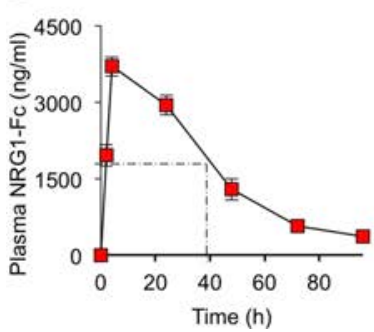

E

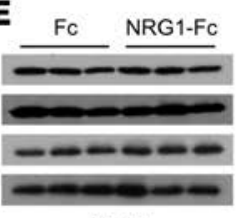

Heart

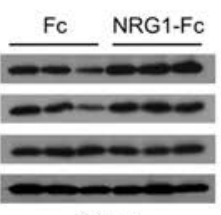

Quad

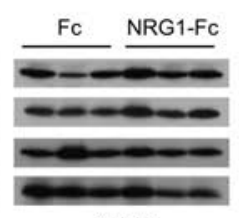

IWAT

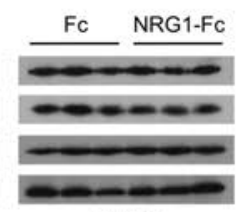

eWAT

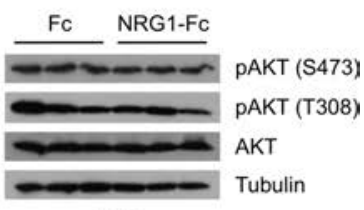

BAT

Figure 1. Generation and characterization of NRG1-Fc fusion protein. (A) A schematic diagram of the NRG1-Fc construct. Signal sequence (SS) and serine glycine linker (gray) are indicated. (B) Plasma concentrations of NRG1-Fc in mice receiving a single i.p. injection of the fusion protein (0.6 mg/ $\mathrm{kg}, n=9$ ). (C) Immunoblots of total cell lysates from T47D cells treated with different concentrations (nM) of indicated proteins for 15 minutes. (D) Immunoblots of total liver lysates from overnight fasted mice 15 minutes after intravenous injection of $100 \mu \mathrm{g} / \mathrm{kg}$ Fc or NRG1-Fc. (E) Immunoblots of total tissue lysates from treated mice. Data represent mean \pm SEM. Quad, quadriceps muscle; iWAT, inguinal white adipose tissue; eWAT, epididymal white adipose tissue; BAT, brown adipose tissue.

ment lowered blood glucose and reduced weight gain $(18,19)$. However, the physiological and molecular mechanisms through which NRG1 exerts these metabolic effects remain largely unknown. Recombinant NRG1 has been tested in clinical studies to improve cardiac function in heart failure patients (20-22). Due to its short plasma half-life, continuous intravenous administration of recombinant NRG1 was required to achieve therapeutic levels in circulation. In this study, we generated a long-lived NRG1-Fc fusion protein that potently lowers blood glucose and promotes weight loss in high-fat diet-fed (HFD-fed) mice. Our findings revealed unexpected dual effects of recombinant NRG1-Fc on the inhibition of hepatic gluconeogenesis and the central regulation of food intake.

\section{Results}

Recombinant NRG1-Fc triggers potent and selective AKT activation in the liver. The NRG family of ligands has been demonstrated to elicit beneficial metabolic effects in mice. NRG1 engages the ErbB receptor tyrosine kinases through its EGF-like domain, a small fragment that exhibits short half-life in circulation (23). Poor pharmacokinetic properties hinder the assessment of its potential as a therapeutic biologic for improving metabolic health in chronic settings. To address this, we fused the EGF-like domain of human NRG1 (amino acids 176-226) to the Fc fragment of human IgG1 (NRG1-Fc; Figure 1A). This strategy has been successfully used to improve treatment efficacy of therapeutic biologics by increasing their half-life in circulation $(24,25)$. As expected, in mice recombinant NRG1-Fc protein exhibited markedly prolonged plasma half-life, reaching approximately 40 hours following i.p. injection (Figure 1B). NRG1-Fc stimulated tyrosine phosphorylation of ErbB3 and ErbB4 in T47D cells in a dose-dependent manner (Figure 1C). Intriguingly, NRG1-Fc exhibited higher potency in triggering ErbB receptor activation and AKT phosphorylation than a commercial recombinant NRG1 protein that contains the EGF-like domain alone. As the NRG1-Fc fusion protein exists as a dimer as a result of $\mathrm{Fc}$ domain dimerization, our results suggest that the dimeric NRG1 ligand may be more efficacious in receptor engagement and signaling.

We next examined the effects of NRG1-Fc on ErbB signaling in C57BL/6 mice after overnight fasting. Compared with FC control, intravenous injection of NRG1-Fc $(100 \mu \mathrm{g} / \mathrm{kg})$ resulted in rapid induction of endogenous ErbB3 phosphorylation within 15 minutes in the liver, which was accompanied by robust activation of AKT and S6K (Figure 1D). This stimulatory effect was also observed at lower doses $(25 \mu \mathrm{g} / \mathrm{kg}$, data 
A

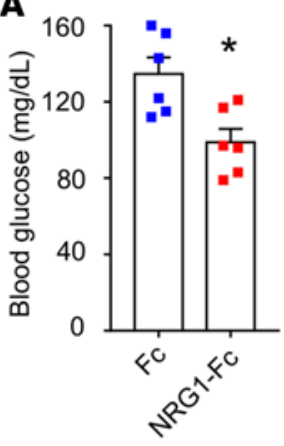

D

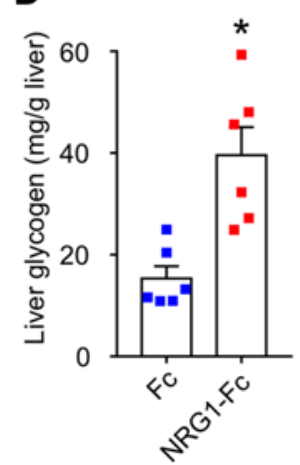

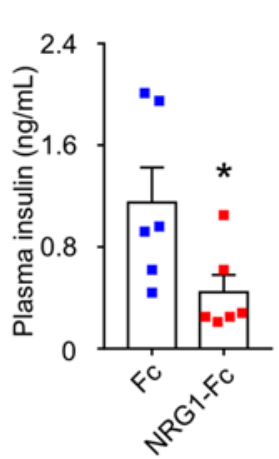
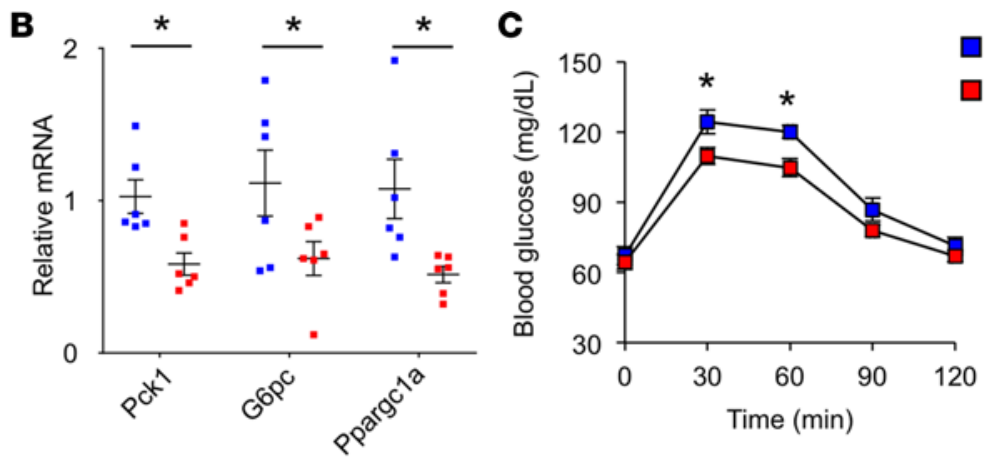

$\mathrm{FC}$

NRG1-FG
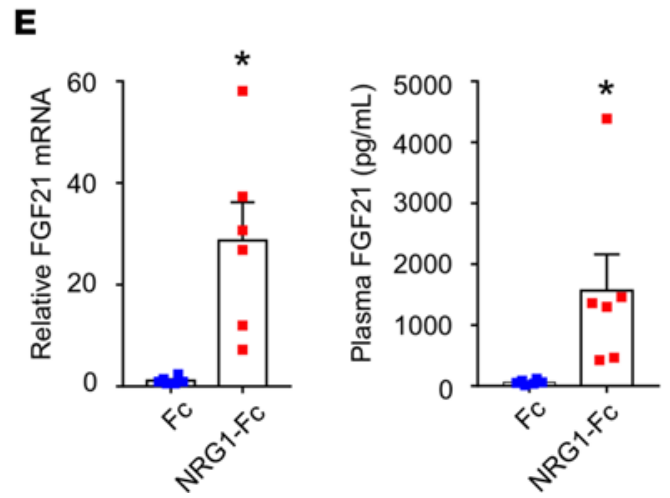

Figure 2. Effects of NRG1-Fc on glucose metabolism in chow-fed mice. (A) Blood glucose and plasma insulin levels in chow-fed mice 6 hours following i.p. injection $(100 \mu \mathrm{g} / \mathrm{kg})$ of $\mathrm{Fc}(n=6)$ or NRG1-Fc $(n=6)$. (B) qPCR analysis of hepatic gene expression in treated mice. (C) Pyruvate tolerance test in chow-fed mice 1 day after Fc $(n=7)$ or NRG1-Fc $(n=8)$ treatment. (D) Liver glycogen content. (E) Hepatic FGF21 mRNA expression and plasma FGF21 concentrations in mice described in $\mathbf{A}$. Data represent mean \pm SEM. ${ }^{*} P<0.05$, vs. Fc, 2-tailed unpaired Student's $t$ test.

not shown). Similar to cell culture studies, NRG1-Fc was more potent in stimulating ErbB/AKT signaling than recombinant human NRG1 (rhNRG1). In striking contrast, no significant changes in AKT phosphorylation were observed in the heart, quadriceps muscle, inguinal white adipose tissue, epididymal white adipose tissue, and brown adipose tissue following NRG1-Fc treatment (Figure 1E). This remarkable selectivity of hepatic AKT activation by NRG1-Fc demonstrates that, among the peripheral insulin-sensitive metabolic tissues, the liver is likely a primary target of NRG1-Fc action.

NRG1-Fc lowers blood glucose by suppressing hepatic gluconeogenesis. AKT is a key mediator of insulin signaling that suppresses hepatic gluconeogenesis during the postprandial phase. We next examined how acute exposure to NRG1-Fc fusion protein affects hepatic and systemic glucose metabolism. Compared with Fc, a single intravenous injection of NRG1-Fc resulted in significantly lower blood glucose 6 hours after treatment (Figure 2A). To determine whether NRG1-Fc may lower blood glucose by augmenting insulin secretion by islet $\beta$ cells, we measured plasma insulin concentrations in treated mice. As shown in Figure 2A, mice treated with NRG1-Fc had significantly lower plasma insulin concentrations than controls, suggesting that lower blood glucose likely precedes reduced insulin secretion. Quantitative real-time PCR (qPCR) analysis of gene expression indicated that mRNA levels of key gluconeogenic genes, including phosphoenolpyruvate carboxykinase 1 (Pck1) and glucose-6-phosphatase (G6pc), and Ppargcla, a transcriptional coactivator that stimulates gluconeogenic gene expression (26), were decreased in the livers from mice treated with NRG1-Fc (Figure 2B). We performed a pyruvate tolerance test to directly assess hepatic glucose production. Compared with control, blood glucose levels were significantly lower in NRG1-Fctreated mice following pyruvate injection, indicative of attenuated hepatic glucose production (Figure 2C). AKT activation is known to promote glycogen storage in the liver. Accordingly, we observed significantly higher liver glycogen content in mice treated with NRG1-Fc compared with those treated with the Fc control (Figure 2D). Hepatic expression of FGF21, a hepatokine with diverse metabolic effects $(27,28)$, was also strongly induced by approximately 30 -fold in response to NRG1-Fc treatment (Figure 2E). Plas- 
A

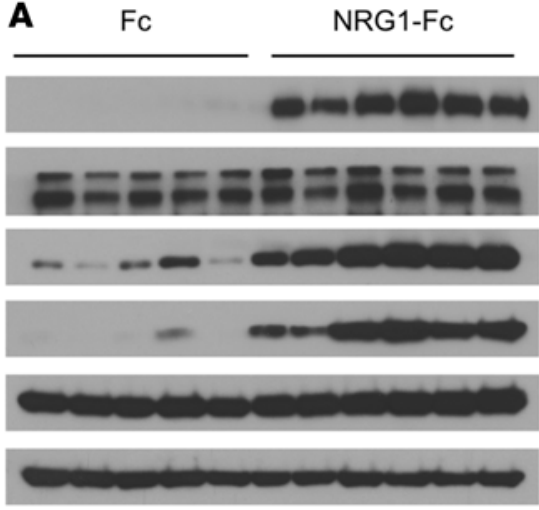

pErbB3 (Y1289)

ErbB3

pAKT (S473)

PAKT (T308)

AKT

Tubulin
C

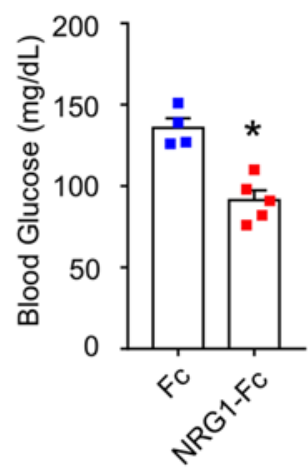

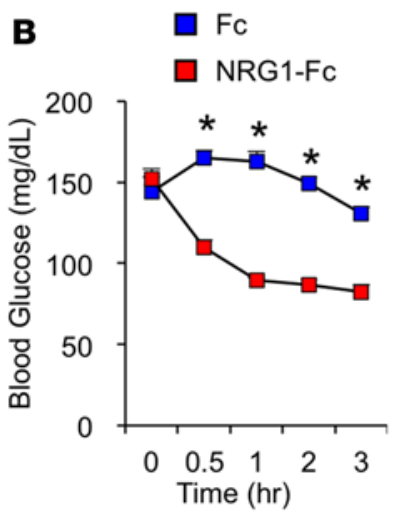
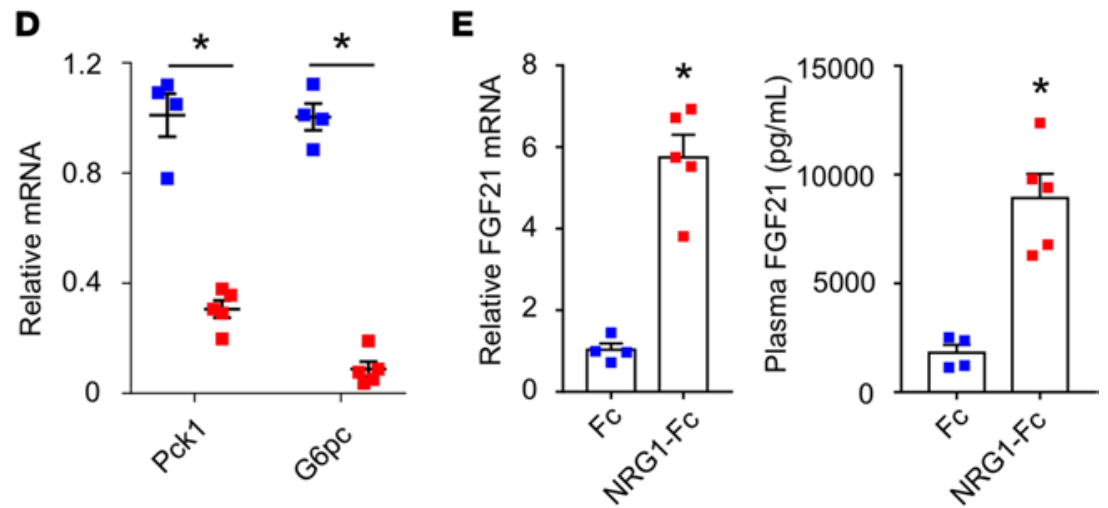

Figure 3. Effects of NRG1-Fc on glucose metabolism in HFD-fed mice. (A) Immunoblots of total liver lysates from mice 4 hours after Fc or NRG1-Fc administration $(100 \mu \mathrm{g} / \mathrm{kg})$. (B) Blood glucose at different time points following a single dose of Fc $(n=7)$ or NRG1-Fc $(n=7)$ in HFD-fed mice. (C) Blood glucose and plasma insulin levels in HFD mice treated with Fc $(n=4)$ or NRG1-Fc $(n=5)$ for 24 hours. (D) qPCR analysis of hepatic gene expression. (E) Hepatic FGF21 mRNA expression and plasma FGF21 concentrations in treated mice. Data represent mean \pm SEM. ${ }^{*} P<0.05$, vs. Fc, 2 -tailed unpaired Student's $t$ test.

ma FGF21 levels reached over 1,500 $\mathrm{pg} / \mathrm{ml}$ compared with approximately $95 \mathrm{pg} / \mathrm{ml}$ in mice treated with $\mathrm{Fc}$, indicating that NRG1-Fc acts as a potent FGF21 secretagogue in mice. The drastic rise of circulating FGF21 by NRG1-Fc diminished 2-3 days following treatment with the fusion protein (data not shown).

We next examined the effects of NRG1-Fc on metabolic signaling and glucose metabolism in HFD-fed obese mice. Similar to the observations in lean mice, we found that tail vein injection of NRG1-Fc potently stimulated ErbB3 and AKT phosphorylation in the liver (Figure 3A). Compared with controls, blood glucose levels were significantly lower in the mice treated with NRG1-Fc 30 minutes after the treatments (Figure 3B). This decrease in blood glucose was sustained 1 day after a single dose of NRG1-Fc, accompanied by lower plasma insulin concentrations (Figure 3C). Hepatic gene expression analysis indicated that Pck1 and G6pc mRNA expression was markedly inhibited by NRG1-Fc (Figure 3D). Hepatic expression and plasma levels of FGF21 were significantly induced by NRG1-Fc in diet-induced obese mice (Figure 3E). Together, these results suggest that reduced hepatic gluconeogenesis likely underlies the acute glucose-lowering effects of NRG1-Fc.

NRG1-Fc resolves key aspects of selective hepatic insulin resistance in diet-induced obese mice. The potent effects of NRG1-Fc on hepatic signaling and glucose metabolism prompted us to investigate the efficacy of chronic administration of NRG1-Fc in ameliorating metabolic disorders associated with obesity. HFD-fed obese mice were i.p. injected biweekly with Fc or NRG1-Fc $(25 \mu \mathrm{g} / \mathrm{kg})$. We measured body weight and food intake of treated mice daily. While mice receiving $\mathrm{Fc}_{\mathrm{c}}$ had modest changes in body weight, the NRG1-Fc-treated group lost approximately $10 \%$ of their body weight within 1 week, accompanied by significantly reduced cumulative food intake during the treatment period (Figure 4, A and B). Plasma triglyceride (TAG) and nonesterified fatty acid (NEFA) concentrations were largely unaffected by recombinant NRG1-Fc (Figure 4C). In contrast, blood glucose and plasma insulin levels were significantly lower in mice treated with NRG1-Fc under both fed and fasted conditions (Figure 4, D and E). Consistently, glucose and insulin tolerance tests demonstrated that NRG1-Fc treatment markedly improved whole-body glucose tolerance and insulin sensitivity in obese mice 
A

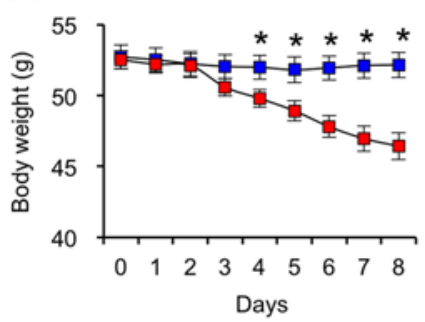

D

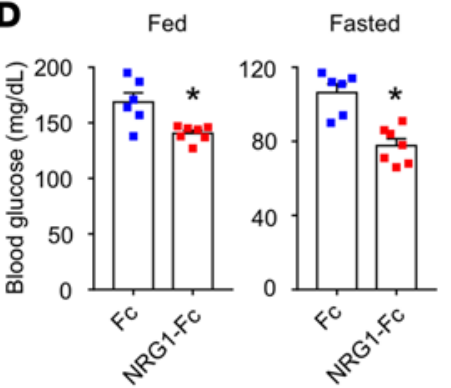

$\mathbf{F}$

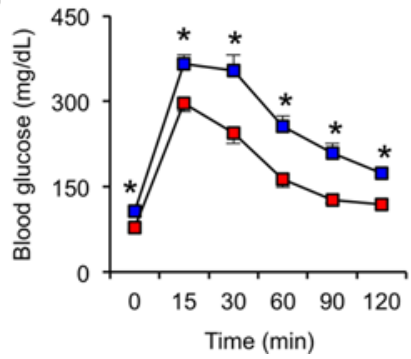

B

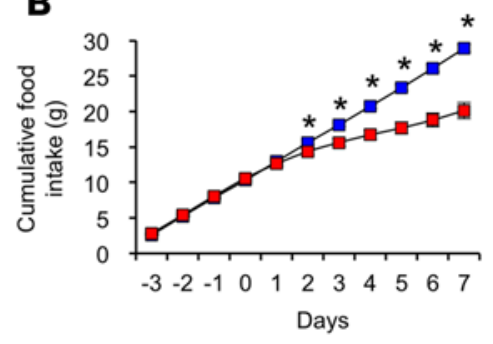

E
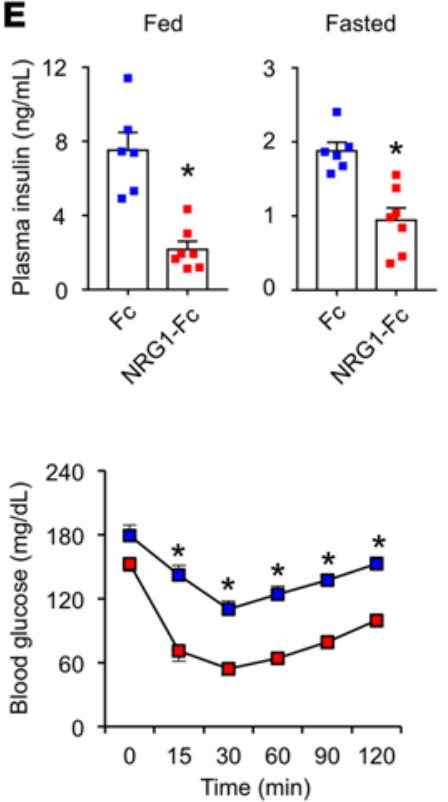

C
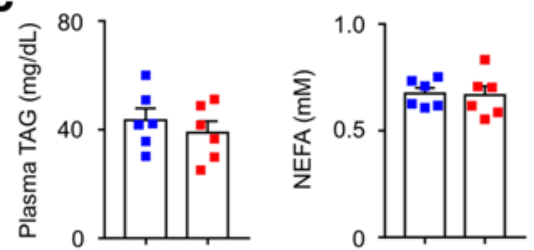

- Fc

DRG1-Fc
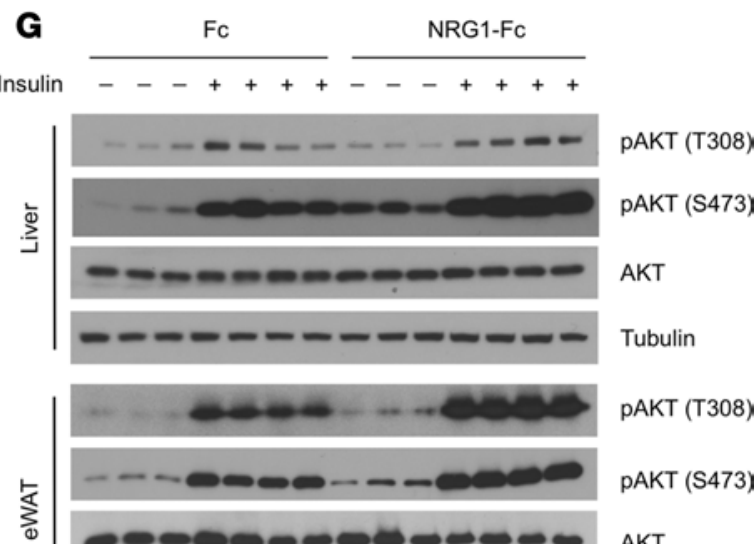

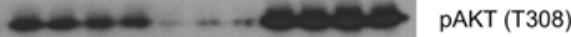

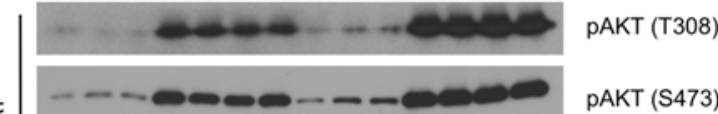

$\aleph_{0}$

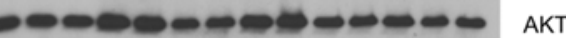

Tubulin

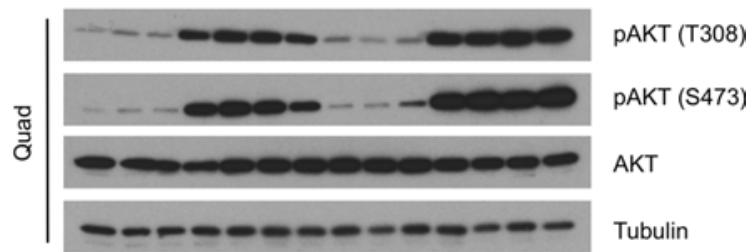

Figure 4. Chronic effects of NRG1-Fc on energy balance and insulin sensitivity. (A) Body weight and (B) cumulative food intake in HFD-fed mice treated with Fc $(n=8)$ or NRG1-Fc $(n=8)$. (C) Plasma TAG and NEFA concentrations in treated mice. (D) Blood glucose and (E) plasma insulin levels in HFD-fed mice treated with Fc $(n=6)$ or NRG1-Fc $(n=6)$ measured under fed and fasted conditions. (F) Glucose tolerance test (left) and insulin tolerance test (right). (C) Immunoblots of total liver, epididymal white adipose tissue (eWAT), and quadriceps muscle (Quad) lysates 15 minutes following intravenous insulin injection. Data represent mean \pm SEM. ${ }^{*} P<0.05$, vs. Fc, 2-tailed unpaired Student's $t$ test (C-E) or 2-way ANOVA (A, B, and F).

(Figure 4F). To directly assess insulin sensitivity in peripheral tissues, we injected HFD-fed mice treated with Fc or NRG1-Fc for 7 days with a single dose of insulin via the tail vein and examined AKT activation in the liver, epididymal white adipose tissue, and quadriceps muscle. As expected, insulin robustly activated AKT phosphorylation in these insulin-responsive tissues (Figure 4G). Insulin-stimulated AKT phosphorylation was further augmented by pretreatment of mice with recombinant NRG1-Fc fusion protein.

Obesity is commonly linked to aberrant activation of both gluconeogenesis and de novo lipogenesis in the liver, a phenomenon described as selective hepatic insulin resistance (29). As a result, elevated hepatic glucose output contributes to hyperglycemia in diabetes, whereas increased lipogenesis exacerbates hepatic steatosis and hyperlipidemia. We next examined whether NRG1-Fc treatment ameliorates the molecular features associated with selective hepatic insulin resistance. We found that, compared with control mice treated with Fc, mRNA levels of gluconeogenic genes (Pck1 and G6pc) were significantly lower in the livers from mice treated with NRG1-Fc under fasted conditions (Figure 5A). The expression of several genes involved in de novo lipogenesis, including acetyl-CoA carboxylase 2 (Acc2), malic enzyme 1 (Me1), stearoyl-CoA desaturase 1 (Scd1), and thyroid hormone responsive (Thrsp, also known as S14), was also attenuated. Under fed conditions, the expression of a larger set of lipogenic genes, including Acc2, Me1, ATP-citrate lyase (Acly), fatty acid synthase (Fasn), Scd1, Thrsp, and cell death-inducing DFFA-like effector c (Cidec, also known as Fsp27), was significantly lower in NRG1-Fc-treated mice (Figure 5B). The expression of genes involved in inflammatory signaling, including Tnfa, C-C motif chemokine 2 (Ccl2), and adhesion G protein-coupled receptor E1 (Adgre1, also known as F4/80), remained largely unaffected 

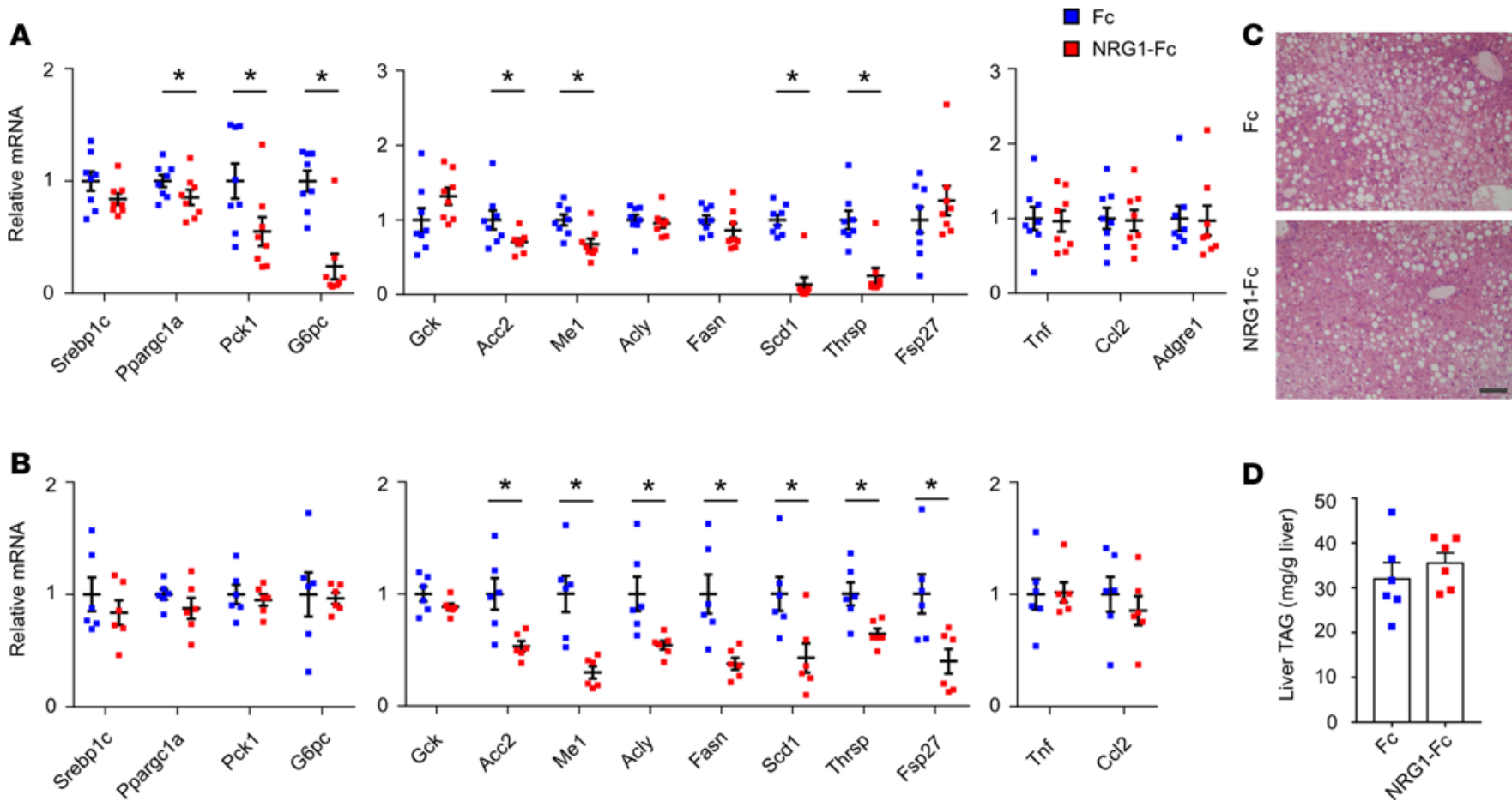

Figure 5. Effects of chronic NRG1-Fc administration on hepatic gene expression and steatosis. (A) qPCR analysis of hepatic gene expression in HFD-fed mice treated with Fc $(n=8)$ or NRG1-Fc $(n=8)$ and analyzed under fasted condition. (B) qPCR analysis of hepatic gene expression in HFD-fed mice treated with Fc $(n=6)$ or NRG1-Fc $(n=6)$ and analyzed under fed conditions. (C) H\&E staining of liver sections. Scale bar: $100 \mu \mathrm{m}$. (D) Liver TAG content. Data represent mean $\pm \mathrm{SEM}$. ${ }^{*} P<0.05$, vs. Fc, 2 -tailed unpaired Student's $t$ test.

by the treatments. As such, chronic NRG1-Fc treatment exerts an inhibitory effect on transcriptional activation of hepatic gluconeogenic and lipogenic gene programs in obese mice. Surprisingly, NRG1-Fc had modest effects on hepatic fat content (Figure 5, C and D). As mice treated with NRG1-Fc had markedly lower plasma insulin levels, it is likely that increased adipose tissue lipolysis may offset the beneficial effects of lipogenic inhibition on hepatic steatosis.

Direct action of NRG1 in POMC neurons mediates its inhibitory effects on food intake. FGF21 is a hepatokine that has been demonstrated to promote energy expenditure and improve insulin sensitivity $(27,28)$. The robust increase of plasma FGF21 levels following NRG1-Fc treatment raised the possibility that FGF21 may mediate certain metabolic effects of NRG1. To test this, we administered recombinant Fc or NRG1-Fc protein into HFD-fed control and liver-specific FGF21-knockout (LKO) mice. As expected, plasma FGF21 levels were elevated in the control group following NRG1-Fc treatment (Figure 6A, $P=0.07$ ). FGF21 was nearly undetectable in plasma from LKO mice, consistent with previous findings that the liver is the major source of FGF21 in circulation (30). To our surprise, NRG1-Fc treatment resulted in similar weight loss and glucose lowering in control and LKO mice (Figure 6, B and C). Further, the inhibitory effect of NRG1Fc on food intake was largely unaffected by FGF21 deficiency (Figure 6D). As such, while we cannot rule out that certain functions of NRG1 may require FGF21, the latter appears to be largely dispensable for the effects of NRG1-Fc on energy balance and glucose metabolism.

To explore the neural mechanisms by which NRG1-Fc suppresses food intake, we examined whether NRG1-Fc directly acts on the hypothalamus to trigger neuronal activation. ErbB4, but not ErbB3, is abundantly expressed in the central nervous system $(31,32)$. Intravenous administration of NRG1-Fc robustly stimulated ErbB4 phosphorylation in the hypothalamus (Figure 7A). Accordingly, mRNA expression of c-Fos, a molecular marker of neuronal activity, was significantly induced in response to NRG1-Fc (Figure 7B). These results demonstrate that circulating NRG1-Fc is capable of penetrating the blood-brain barrier and directly acts on hypothalamic neurons to regulate feeding behaviors.

We next examined the effects of NRG1-Fc on excitability of pro-opiomelanocortin (POMC) neurons in the arcuate nucleus of the hypothalamus (ARH), which are well known to produce anorexigenic effects 
A

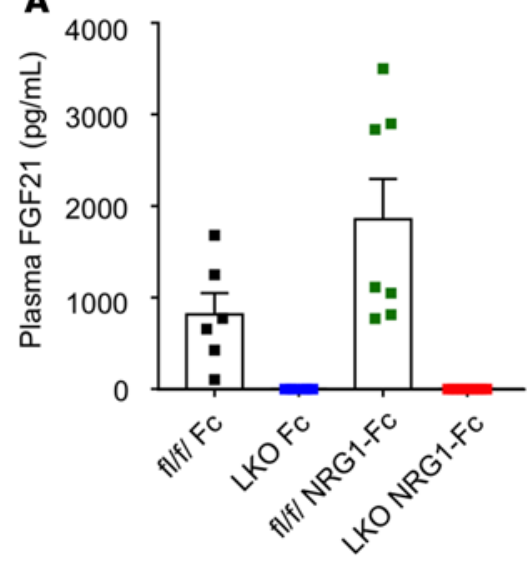

B

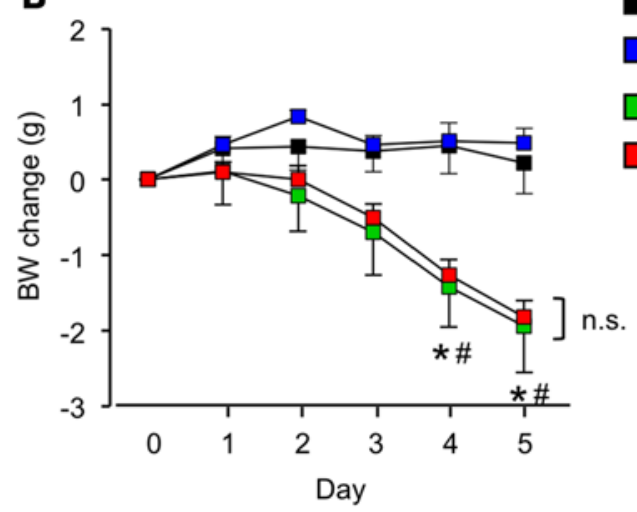

fl/fl Fc, $n=6$

LKO Fc, $n=6$

fl/fl NRG1-Fc, n=7

LKO NRG1-Fc, $n=9$
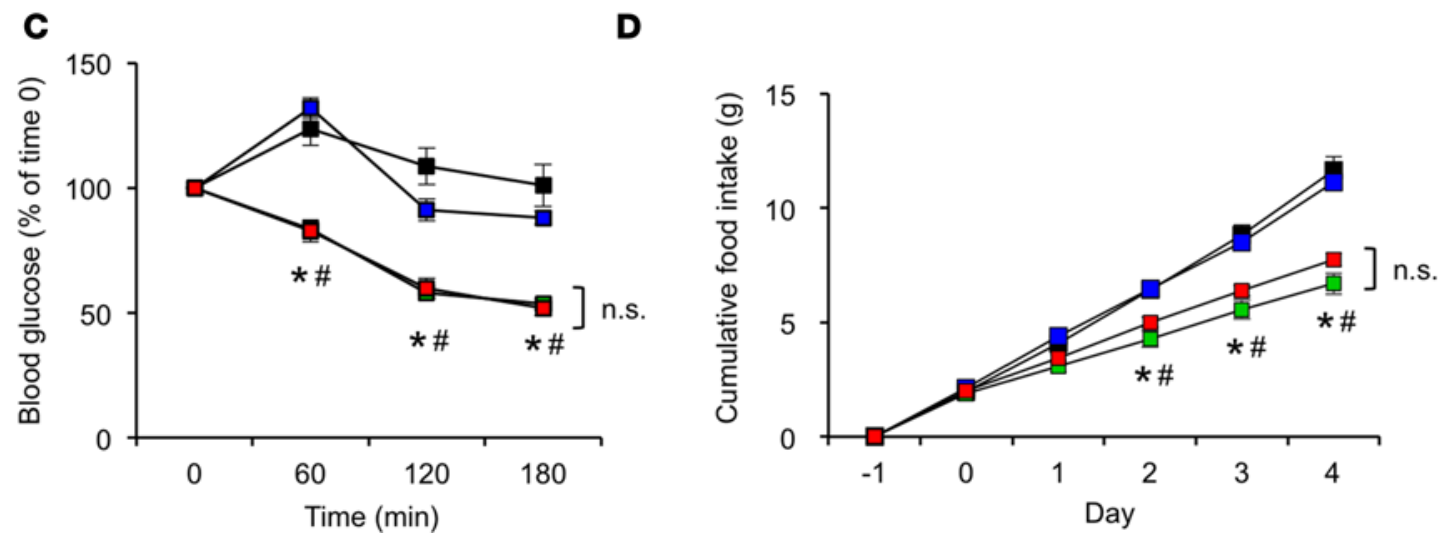

Figure 6. Metabolic effects of NRG1-Fc in liver-specific FGF21-knockout mice. (A) Plasma FGF21 levels in HFD-fed control (fl/fl) and FGF21 LKO mice after Fc or NRG1-Fc treatment, as indicated. (B) Changes in body weight, (C) blood glucose, and (D) cumulative food intake in treated mice. Data represent mean $\pm \mathrm{SEM} .{ }^{*} P<0.05$, NRG1-Fc vs. Fc in LKO mice; ${ }^{\sharp} P<0.05$, NRG1-Fc vs. Fc in $\mathrm{fl} / \mathrm{fl}$ mice, 2 -way ANOVA with multiple comparisons. AUC was evaluated by 2-tailed unpaired Student's $t$ test.

(33). We found that vehicle treatment $(\mathrm{Fc})$ did not affect the resting membrane potential (RM) and firing rate of ARH POMC neurons (Figure 7, C-E). Interestingly, exposure to NRG1-Fc (300 nM, 1-second puff) significantly depolarized 16 of 31 (51.61\%) POMC neurons, while the other 15 POMC neurons tested were not affected. The depolarization was associated with significant increases in firing rate (Figure 7F). As leptin also depolarizes POMC neurons to exert anorectic effects (34), we examined leptin-induced depolarization in TOMATO-labeled mature POMC neurons and found that 13 of 38 (34.21\%) POMC neurons were depolarized by leptin (Figure 7, C and D). Notably, leptin-induced depolarization and increases in firing rate were comparable to responses evoked by NRG1-Fc (Figure 7, E and F). We next recorded NRG1-induced changes in RM in the presence of a cocktail of neuronal inhibitors containing $1 \mu \mathrm{M}$ tetrodotoxin, which blocks action potentials, and a mixture of fast synaptic inhibitors (GABA receptor antagonist, bicuculline; AP-5, NMDA receptor antagonist; CNQX, AMPA receptor antagonist), which block the majority of presynaptic inputs. Under this condition, we found that 9 of 18 (50\%) POMC neurons were depolarized in response to NRG1-Fc (Figure 7, C-E), suggesting that NRG1-Fc likely acts directly upon POMC neurons to modulate their excitability.

\section{Discussion}

NRGs are emerging as an important family of ligands that regulate diverse aspects of glucose and lipid metabolism and energy balance. Nrg4 protects mice from HFD-induced insulin resistance and hepatic steatosis by attenuating de novo lipogenesis and promoting fuel oxidation in the liver. Further, hepatic Nrg4 signaling serves as an endocrine checkpoint for the steatosis-to-NASH transition by protecting hepatocytes from stress-induced cell death. NRG1 improves whole body glucose metabolism and has been shown to exert bene- 
A
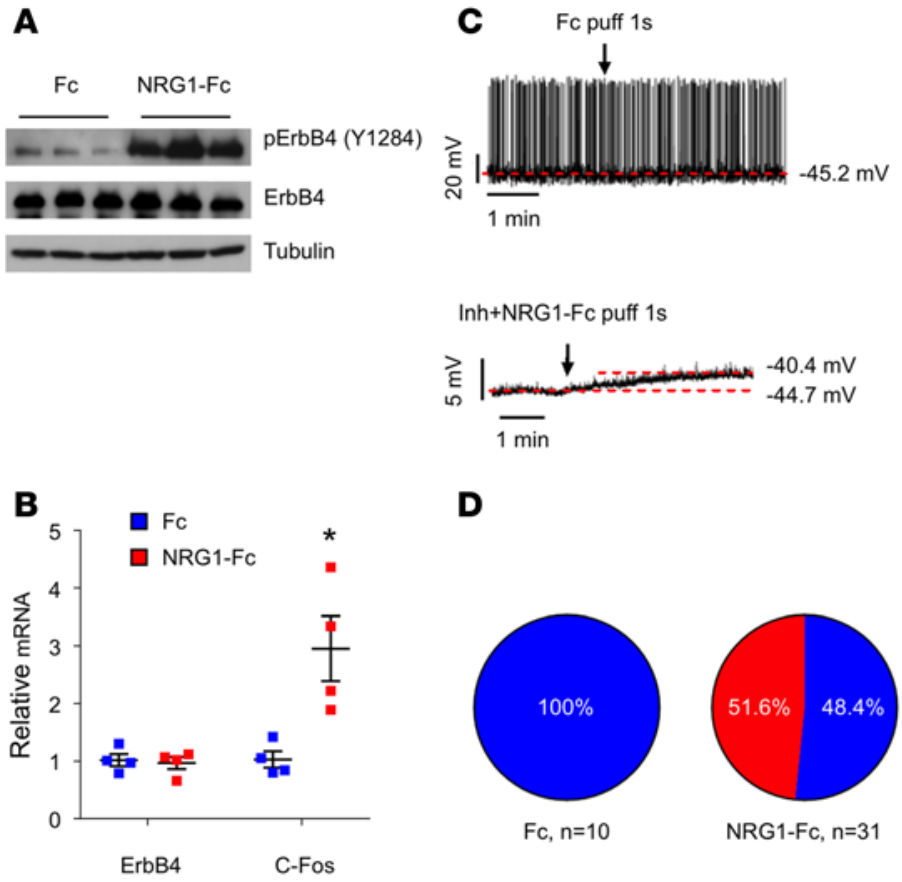

D

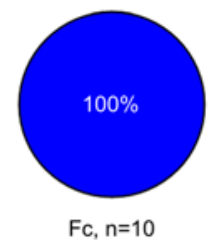

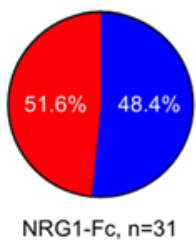
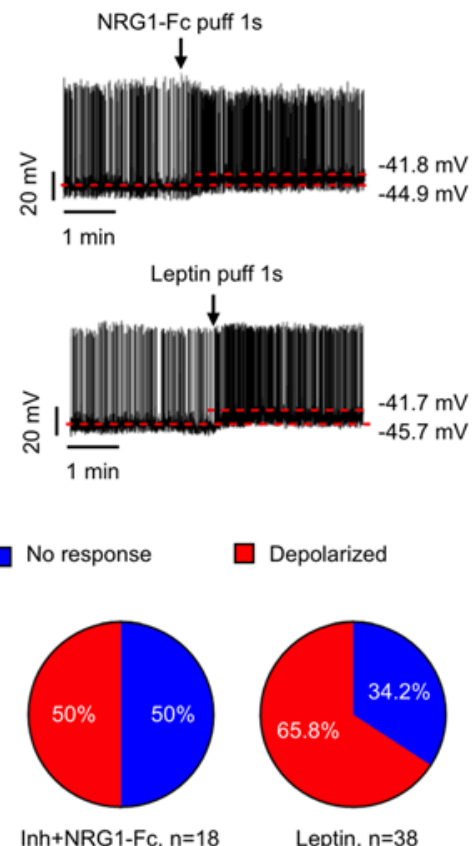

E

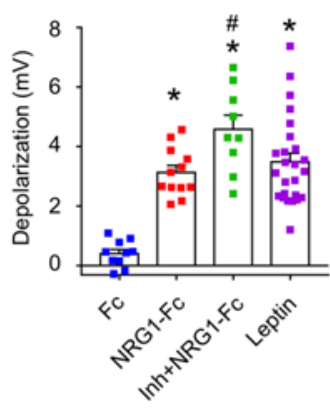

$\mathbf{F}$

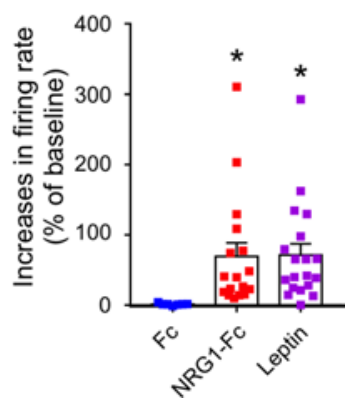

Figure 7. Direct effects of NRG1-Fc on POMC neurons. (A) Immunoblots of hypothalamic lysates from mice treated with Fc or NRG1-Fc (300 $\mu \mathrm{g} / \mathrm{kg})$ for 30 minutes. (B) qPCR analysis of hypothalamic gene expression from mice treated with Fc $(n=4)$ or NRG1-Fc $(n=4)$ for 45 minutes. (C) Representative traces of POMC neurons responding to a 1-second puff of Fc, NRG1-Fc (300 nM), NRG1-Fc in the presence of synaptic inhibitors (tetrodotoxin [TTX] + AP5 + CNQX + bicuculline) in infusion buffer (Inh + NRG1-Fc), or leptin (300 nM). (D) Proportion of cells recorded that responded with depolarization $>2 \mathrm{mV}$ (red) or with no change in resting membrane potential (blue) following the indicated treatments. (E and $\mathbf{F}$ ) (E) Depolarization and (F) increase in firing rate of POMC neurons after the above treatments. Data represent mean \pm SEM. ${ }^{*} P<0.05$ vs. Fc, 2 -tailed unpaired Student's $t$ test $(B) ;{ }^{*} P<0.05$, ${ }^{\#} P<0.05$ vs. NRG1-Fc, 1-way ANOVA with multiple comparisons (E and $\mathbf{F})$.

ficial effects on cardiac function in heart failure patients. Despite these intriguing observations, the therapeutic potential of NRGs remains elusive given the poor plasma half-life of the native ligands. In this study, we generated an NRG1-Fc fusion protein with prolonged plasma half-life and characterized its metabolic action in mice in acute and chronic settings. Our findings revealed surprisingly potent dual effects of recombinant NRG1-Fc on the inhibition of hepatic gluconeogenesis and the central regulation of food intake (Figure 8).

The ErbB receptors, particularly ErbB4, exhibit a broad pattern of tissue distribution, including skeletal muscle and heart (2). As such, the highly selective AKT activation in the liver, but not in other peripheral tissues known to express these ErbB receptors, came as a surprise. In fact, we failed to detect changes in AKT phosphorylation in skeletal muscle and the heart in response to intravenous injection of NRG1-Fc. In contrast, ErbB3/AKT signaling was markedly stimulated by NRG1-Fc in both lean and obese mouse livers, leading to suppression of gluconeogenic gene expression and hepatic glucose output. Following chronic treatments, mice receiving NRG1-Fc had greatly improved insulin sensitivity and glucose tolerance. This improved glucose homeostasis is linked to attenuated expression of genes involved in hepatic gluconeogenesis and de novo lipogenesis. Aberrant activation of these metabolic pathways contributes to hyperglycemia, hyperlipidemia, and hepatic steatosis in metabolic syndrome. Our results suggest that pharmacological activation of NRG1/ErbB signaling may resolve this deleterious metabolic reprogramming in obesity and improve metabolic health. NRGs and other EGF-like ligands are expressed in diverse tissues. Future work should address their role in ErbB-mediated metabolic signaling under various physiological and pathophysiological conditions.

An unexpected finding here is that NRG1-Fc potently stimulated hepatic expression and plasma levels of FGF21. The ability of NRG1-Fc to markedly elevate plasma FGF21 beyond its physiological levels demonstrates that NRG1-Fc serves as a powerful secretagogue for FGF21. Surprisingly, mice lacking FGF21 in the liver exhibited very low levels of plasma FGF21, yet their response to NRG1Fc was nearly indistinguishable from that of control mice. These results suggest that FGF21 is large- 


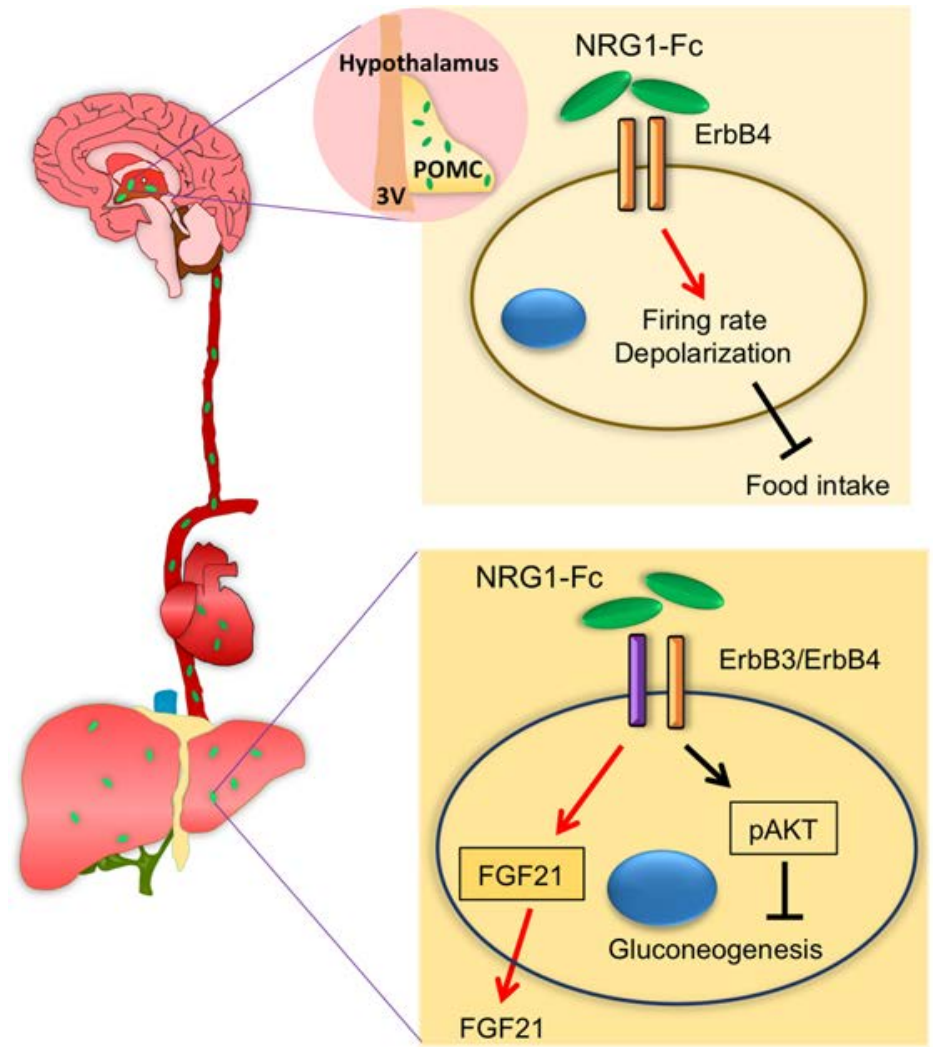

Figure 8. Model depicting the pleiotropic effects of NRG1-Fc in hepatocytes and POMC neurons. NRG-Fc lowers blood glucose by activating hepatic AKT signaling to inhibit gluconeogenesis, while suppressing food intake by depolarizing and increasing the firing rate of POMC neurons.

ly dispensable for the effects of NRG1-Fc on blood glucose and energy balance. It remains unknown, however, whether other aspects of NRG1 action require FGF21 signaling. The inhibitory effects of NRG1-Fc on food intake and body weight are consistent with its direct action in the POMC neurons. In fact, ErbB4 is abundantly expressed in the central nervous system $(31,32)$. Peripheral administration of NRG1-Fc leads to strong ErbB4 phosphorylation and c-Fos induction in the hypothalamus. Remarkably, the NRG1$\mathrm{Fc}$-induced depolarization and increase in firing rate were comparable to responses evoked by leptin, a potent anorexigenic hormone. As NRG1 is highly expressed in the central nervous system (2), it is possible that local NRG1 signaling in the brain may modulate food intake and energy balance. In summary, pharmacological activation of NRG1/ErbB signaling by NRG1-Fc elicits multiple metabolic benefits on blood glucose and body weight, providing proof-of-principle validation for developing biologic therapeutics targeting the NRG/ErbB pathway.

\section{Methods}

Construction and purification of NRG1-Fc fusion protein. The NRG1-Fc fusion construct contains an N-terminal signal peptide from azurocidin 1 followed by the EGF-like domain of human NRG1 (amino acids 176-226 of hNRG1 $\beta$ ), a glycine-serine linker and human IgG1 Fc fragment (Figure 1A). The construct was synthesized by GeneArt (Thermo Fisher Scientific) and subcloned into pcDNA3 expression vector. For fusion protein production, the Fc vector and NRG1-Fc constructs were transiently transfected into suspension HEK293 cells using the Expi293 Expression System (Thermo Fisher Scientific). Conditional media were collected 7 days after transfection, adjusted to the composition of binding buffer $(0.2 \mathrm{M}$ sodium phosphate, $\mathrm{pH} 7.0)$, filtered through a $0.45-\mu \mathrm{m}$ filter (Millipore), and processed for affinity purification using a Hitrap rProtein A FF $5 \mathrm{ml}$ column on the ÄKTA Pure FPLC chromatography system (GE Healthcare). The column was washed with $50 \mathrm{ml}$ binding buffer and eluted with a $\mathrm{pH}$ 3-7 gradient elution buffer ( $0.1 \mathrm{M}$ sodium citrate, $\mathrm{pH}$ 3.0). Fusion proteins were dialyzed in $1 \times$ phosphate-buffered saline buffer $\left(137 \mathrm{mM} \mathrm{NaCl}, 2.7 \mathrm{mM} \mathrm{KCl}, 10 \mathrm{mM} \mathrm{Na} \mathrm{H}_{-}\right.$ PO4, $1.8 \mathrm{mM} \mathrm{KH}_{2} \mathrm{PO} 4, \mathrm{pH}$ 7.4) using a Slide-A-Lyzer Dialysis Cassette (Thermo Fisher Scientific).

Mouse studies. Mice were maintained under 12-hour-light/dark cycles, with free access to food and water. Teklad 5001 laboratory diet was used as standard chow. For HFD feeding, mice were fed a diet containing $60 \%$ of calories from fat (D12492, Research Diets). C57BL/6J mice were obtained from The Jackson Laboratory. The generation of LKO mice was previously described (30).

For measurements of NRG1-Fc plasma half-life, male C57BL/6J mice received an i.p. injection of NRG1-Fc $(0.6 \mathrm{mg} / \mathrm{kg})$. NRG1-Fc fusion protein was quantified using the Human IgG ELISA Quantitation Set (Bethyl Laboratories Inc.). Glucose, insulin, and pyruvate tolerance tests were performed as previously described $(35,36)$. For insulin signaling studies, HFD-fed mice received 3 doses of Fc or NRG1-Fc $(50 \mu \mathrm{g} /$ $\mathrm{kg} \mathrm{BW})$ over a period of 1 week and were fasted for 16 hours before intravenous insulin injection $(1.5 \mathrm{U} / \mathrm{kg}$ $\mathrm{BW})$. Tissues were harvested 10 minutes after injection insulin for immunoblotting analyses.

Liver glycogen and TAG were measured as previously described (35, 37). Plasma concentrations of TAG and NEFA were measured using Serum Triglyceride Determination Kit (TR0100 SIGMA) and Free Fatty Acid Quantitation Kit (MAK044 SIGMA), respectively. Plasma insulin and FGF21 concentrations were measured using a Mouse Insulin ELISA Kit (Crystal Chem) and mouse/rat FGF21 Quantikine ELISA Kit (R\&D Systems), respectively. 
Electrophysiology. POMC-CreER/Rosa26-tdTOMATO mice received tamoxifen inductions $(0.2 \mathrm{mg} / \mathrm{g}$, i.p., twice at 9 weeks of age). At 13-14 weeks of age, these mice were deeply anesthetized with isoflurane at 9:00-9:30 am and transcardially perfused with modified ice-cold artificial cerebral spinal fluid (aCSF; 10 $\mathrm{mM} \mathrm{NaCl}, 25 \mathrm{mM} \mathrm{NaHCO}_{3}, 195 \mathrm{mM}$ sucrose, $5 \mathrm{mM}$ glucose, $2.5 \mathrm{mM} \mathrm{KCl}, 1.25 \mathrm{mM} \mathrm{NaH}_{2} \mathrm{PO}_{4}, 2 \mathrm{mM}$ $\mathrm{Na}$ pyruvate, $0.5 \mathrm{mM} \mathrm{CaCl}_{2}, 7 \mathrm{mM} \mathrm{MgCl}$ ). The mice were then decapitated, and the entire brain was removed. Brains were quickly sectioned in ice-cold aCSF solution $(126 \mathrm{mM} \mathrm{NaCl}, 2.5 \mathrm{mM} \mathrm{KCl}, 1.2 \mathrm{mM}$ $\mathrm{MgCl}_{2}, 2.4 \mathrm{mM} \mathrm{CaCl}_{2}, 1 \mathrm{mM} \mathrm{NaH}_{2} \mathrm{PO}_{4}, 11.1 \mathrm{mM}$ glucose, and $21.4 \mathrm{mM} \mathrm{NaHCO}_{3}$ ) saturated with $95 \% \mathrm{O}_{2}$ and $5 \% \mathrm{CO}_{2}$. Coronal sections containing the $\mathrm{ARH}(250 \mu \mathrm{m})$ were cut with a Microm HM 650V vibratome (Thermo Scientific). Then, the slices were recovered in the aCSF at $34^{\circ} \mathrm{C}$ for 1 hour.

Whole-cell patch clamp recordings were performed in the TOMATO-labeled mature POMC neurons in the ARH visually identified by an upright microscope (Eclipse FN-1, Nikon) equipped with IR-DIC optics (Nikon $\times 40$ NIR). Signals were processed using a Multiclamp 700B amplifier (Axon Instruments), sampled using Digidata 1440A, and analyzed offline on a PC with pCLAMP 10.3 (Axon Instruments). The slices were bathed in oxygenated aCSF $\left(32^{\circ} \mathrm{C}-34^{\circ} \mathrm{C}\right)$ at a flow rate of approximately $2 \mathrm{ml} / \mathrm{min}$. Patch pipettes with resistances of 3-5 $\mathrm{M} \Omega$ were filled with solution containing $126 \mathrm{mM} \mathrm{K}$ gluconate, $10 \mathrm{mM}$ $\mathrm{NaCl}, 10 \mathrm{mM}$ EGTA, $1 \mathrm{mM} \mathrm{MgCl}$, 2 mM Na-ATP, and $0.1 \mathrm{mM} \mathrm{Mg-GTP}$ (adjusted to $\mathrm{pH} 7.3$ with $\mathrm{KOH}$ ).

Current clamp was engaged to test neural firing frequency and RM at the baseline and after puff application of vehicle, NRG1, leptin (300 nM, 1-second puff). In some experiments, the aCSF solution also contained $1 \mu \mathrm{M}$ tetrodotoxin and a cocktail of fast synaptic inhibitors, namely bicuculline ( $50 \mu \mathrm{M}$; a GABA receptor antagonist), AP-5 (30 $\mu \mathrm{M}$; an NMDA receptor antagonist), and CNQX (30 $\mu \mathrm{M}$; an AMPA receptor antagonist), to block the majority of presynaptic inputs. The values for RM and firing frequency were averaged within 2-minute bin at the baseline or after puffs. The RM values were calculated by Clampfit 10.3 using the "analysis statistic" function of the software. A neuron was considered depolarized or hyperpolarized if a change in membrane potential was at least $2 \mathrm{mV}$ in amplitude and this response was observed after leptin application and stayed stable for at least 2 minutes. At the end of recordings, lucifer yellow dye was included in the pipette solution to trace the recorded neurons, and the brain slices were fixed with $4 \%$ formalin overnight and mounted onto slides. Cells were then visualized with a Leica DM5500 fluorescence microscope to identify post hoc the anatomical location of the recorded neurons in the ARH.

Gene expression analysis. Gene expression analysis was performed as previously described (35, 38). Briefly, total RNA was isolated using the TRIzol method. For qPCR analysis, $2 \mu \mathrm{g}$ total RNA was reverse-transcribed using MMLV-RT (Invitrogen) and quantified by the QuantStudio 6 Flex Real-Time PCR System using SYBR Green (Thermo Fisher Scientific). Relative mRNA expression was normalized to the levels of ribosomal protein 36B4.

Immunoblotting analyses. T47D cells were maintained in DMEM containing 10\% FBS and starved for 12-16 hours in serum-free DMEM before treatments with Fc, NRG1-Fc, or rhNRG1 (R\&D Systems) for 15 minutes. Total cell lysates were prepared in a lysis buffer containing $50 \mathrm{mM}$ Tris $(\mathrm{pH} 7.5), 150 \mathrm{mM}$ $\mathrm{NaCl}, 5 \mathrm{mM}$ NaF, $25 \mathrm{mM} \beta$-glycerol-phosphate, $1 \mathrm{mM}$ sodium orthovanadate, $10 \%$ glycerol, $1 \%$ Triton $\mathrm{X}-100,1 \mathrm{mM}$ dithiothreitol, and freshly added protease inhibitors. Protein concentrations were determined by the BCA method using Protein Assay Dye Reagent Concentrate (Bio-Rad). Antibodies used in this study were: phospho-ErbB3 (Y1289, catalog 4791), phospho-ErbB4 (Y1284, catalog 4757), ErbB4 (catalog 4795), phospho-AKT (T308, catalog 4056), phospho-AKT (S473, catalog 4058), AKT (catalog 4691), phospho-S6K (catalog 9234), and S6K (catalog 2708) (Cell Signaling Technology); Tubulin (T6199, MilliporeSigma); and ErbB3 (sc-285, Santa Cruz Biotechnology).

Statistics. Statistical analysis was performed using GraphPad Prism 7. Statistical differences were evaluated using 2-tailed unpaired Student's $t$ test or 1-way or 2-way ANOVA with multiple comparisons, as indicated. $P$ values of less than 0.05 were considered statistically significant.

Study approval. All mouse studies were performed according to procedures approved by the University Committee on Use and Care of Animals at the University of Michigan, Ann Arbor, Michigan, USA.

\section{Author contributions}

JDL, PZ, and HK conceived the project and designed the research. PZ, HK, YH, SOI, SL, ZC, ZY, and XC performed the experiments. PZ, HK, JDL, YX, SOI, MJP, and KZ analyzed the data. PZ, HK, and JDL wrote and reviewed the manuscript. 


\section{Acknowledgments}

This work is supported by the NIH (DK102456 and AG055379 to JDL; DK101379 and DK117281 to YX; DK106104 to MJP), the American Heart Association (17GRNT32960003 to YX), the United States Department of Agriculture/Current Research Information System (3092-5-001-059 to YX); and the American Diabetes Association (1-17-PDF-138 to YH). HK is supported by a T32 Medical Scientist Training Program fellowship (NIH GM007863).

Address correspondence to: Jiandie Lin, 5437 Life Sciences Institute, University of Michigan, 210 Washtenaw Avenue, Ann Arbor, Michigan 48109, USA. Phone: 734.615.3512; Email: jdlin@umich.edu.

1. Burgess AW. EGFR family: structure physiology signalling and therapeutic targets. Growth Factors. 2008;26(5):263-274.

2. Falls DL. Neuregulins and the neuromuscular system: 10 years of answers and questions. J Neurocytol. 2003;32(5-8):619-647.

3. Schneider MR, Wolf E. The epidermal growth factor receptor ligands at a glance. J Cell Physiol. 2009;218(3):460-466.

4. Salinas YD, Wang L, DeWan AT. Multiethnic genome-wide association study identifies ethnic-specific associations with body mass index in Hispanics and African Americans. BMC Genet. 2016;17(1):78.

5. Lee M, Kwon DY, Kim MS, Choi CR, Park MY, Kim AJ. Genome-wide association study for the interaction between BMR and BMI in obese Korean women including overweight. Nutr Res Pract. 2016;10(1):115-124.

6. Chen Z, et al. Nrg4 promotes fuel oxidation and a healthy adipokine profile to ameliorate diet-induced metabolic disorders. Mol Metab. 2017;6(8):863-872.

7. Harari D, et al. Neuregulin-4: a novel growth factor that acts through the ErbB-4 receptor tyrosine kinase. Oncogene. 1999;18(17):2681-2689.

8. Rosell M, et al. Brown and white adipose tissues: intrinsic differences in gene expression and response to cold exposure in mice. Am J Physiol Endocrinol Metab. 2014;306(8):E945-E964.

9. Wang GX, et al. The brown fat-enriched secreted factor Nrg4 preserves metabolic homeostasis through attenuation of hepatic lipogenesis. Nat Med. 2014;20(12):1436-1443.

10. Wang GX, Zhao XY, Lin JD. The brown fat secretome: metabolic functions beyond thermogenesis. Trends Endocrinol Metab. 2015;26(5):231-237

11. Cai C, Lin M, Xu Y, Li X, Yang S, Zhang H. Association of circulating neuregulin 4 with metabolic syndrome in obese adults: a cross-sectional study. BMC Med. 2016;14(1):165.

12. Dai YN, et al. A case-control study: Association between serum neuregulin 4 level and non-alcoholic fatty liver disease. Metab Clin Exp. 2015;64(12):1667-1673.

13. Jiang J, et al. Circulating neuregulin 4 levels are inversely associated with subclinical cardiovascular disease in obese adults. $S c i$ Rep. 2016;6:36710.

14. Guo L, et al. Hepatic neuregulin 4 signaling defines an endocrine checkpoint for steatosis-to-NASH progression. J Clin Invest. 2017;127(12):4449-4461.

15. Cantó C, et al. Neuregulins increase mitochondrial oxidative capacity and insulin sensitivity in skeletal muscle cells. Diabetes. 2007;56(9):2185-2193.

16. Cantó C, et al. Neuregulin signaling on glucose transport in muscle cells. J Biol Chem. 2004;279(13):12260-12268.

17. Suárez E, Bach D, Cadefau J, Palacin M, Zorzano A, Gumá A. A novel role of neuregulin in skeletal muscle. Neuregulin stimulates glucose uptake, glucose transporter translocation, and transporter expression in muscle cells. J Biol Chem. 2001;276(21):18257-18264.

18. Ennequin $\mathrm{G}$, et al. Neuregulin 1 affects leptin levels, food intake and weight gain in normal-weight, but not obese, $\mathrm{db} / \mathrm{db}$ mice Diabetes Metab. 2015;41(2):168-172.

19. Ennequin G, et al. Neuregulin 1 Improves Glucose Tolerance in db/db Mice. PLoS One. 2015;10(7):e0130568

20. Gao R, et al. A Phase II, randomized, double-blind, multicenter, based on standard therapy, placebo-controlled study of the efficacy and safety of recombinant human neuregulin-1 in patients with chronic heart failure. J Am Coll Cardiol. 2010;55(18):1907-1914.

21. Jabbour A, et al. Parenteral administration of recombinant human neuregulin-1 to patients with stable chronic heart failure produces favourable acute and chronic haemodynamic responses. Eur J Heart Fail. 2011;13(1):83-92.

22. Mendes-Ferreira $P$, et al. Neuregulin-1 improves right ventricular function and attenuates experimental pulmonary arterial hypertension. Cardiovasc Res. 2016;109(1):44-54.

23. Liu X, et al. Neuregulin-1/erbB-activation improves cardiac function and survival in models of ischemic, dilated, and viral cardiomyopathy. J Am Coll Cardiol. 2006;48(7):1438-1447.

24. Czajkowsky DM, Hu J, Shao Z, Pleass RJ. Fc-fusion proteins: new developments and future perspectives. EMBO Mol Med. 2012;4(10):1015-1028.

25. Wu B, Sun YN. Pharmacokinetics of Peptide-Fc fusion proteins. J Pharm Sci. 2014;103(1):53-64.

26. Liu C, Lin JD. PGC-1 coactivators in the control of energy metabolism. Acta Biochim Biophys Sin (Shanghai). 2011;43(4):248-257.

27. Markan KR, Potthoff MJ. Metabolic fibroblast growth factors (FGFs): Mediators of energy homeostasis. Semin Cell Dev Biol. 2016;53:85-93.

28. Owen BM, Mangelsdorf DJ, Kliewer SA. Tissue-specific actions of the metabolic hormones FGF15/19 and FGF21. Trends Endocrinol Metab. 2015;26(1):22-29.

29. Brown MS, Goldstein JL. Selective versus total insulin resistance: a pathogenic paradox. Cell Metab. 2008;7(2):95-96.

30. Markan KR, et al. Circulating FGF21 is liver derived and enhances glucose uptake during refeeding and overfeeding. Diabetes. 2014;63(12):4057-4063. 
31. Bean JC, et al. Genetic labeling reveals novel cellular targets of schizophrenia susceptibility gene: distribution of GABA and non-GABA ErbB4-positive cells in adult mouse brain. J Neurosci. 2014;34(40):13549-13566.

32. Mei L, Xiong WC. Neuregulin 1 in neural development, synaptic plasticity and schizophrenia. Nat Rev Neurosci. 2008;9(6):437-452.

33. Zhan $\mathrm{C}$, et al. Acute and long-term suppression of feeding behavior by POMC neurons in the brainstem and hypothalamus, respectively. J Neurosci. 2013;33(8):3624-3632.

34. Cowley MA, et al. Leptin activates anorexigenic POMC neurons through a neural network in the arcuate nucleus. Nature. 2001;411(6836):480-484.

35. Meng ZX, et al. Glucose sensing by skeletal myocytes couples nutrient signaling to systemic homeostasis. Mol Cell. 2017;66(3):332-344.e4.

36. Molusky MM, Li S, Ma D, Yu L, Lin JD. Ubiquitin-specific protease 2 regulates hepatic gluconeogenesis and diurnal glucose metabolism through 11ß-hydroxysteroid dehydrogenase 1. Diabetes. 2012;61(5):1025-1035.

37. Li S, et al. Genome-wide coactivation analysis of PGC-1alpha identifies BAF60a as a regulator of hepatic lipid metabolism. Cell Metab. 2008;8(2):105-117.

38. Hernandez C, Molusky M, Li Y, Li S, Lin JD. Regulation of hepatic ApoC3 expression by PGC-1 $\beta$ mediates hypolipidemic effect of nicotinic acid. Cell Metab. 2010;12(4):411-419. 